\title{
Effect of electrical stimulation combined with diet therapy on insulin resistance via mTOR signaling
}

\author{
SIQIN HUANG, NIANZHEN TANG, HONGDI ZHAO and CHENG-LIN TANG \\ Traditional Chinese Medicine College, Chongqing Medical University, Chongqing 400016, P.R. China
}

Received May 27, 2019; Accepted September 18, 2019

DOI: $10.3892 / \mathrm{mmr} .2019 .10783$

\begin{abstract}
Insulin resistance (IR) is the impaired insulin response that causes decreased glucose tolerance. Electrical stimulation (ES) can improve insulin sensitivity in the skeletal muscle. However, the underlying molecular mechanisms remain to be elucidated. In the present study, the effect of ES and diet therapy on IR and the role of the mammalian target of rapamycin (mTOR) pathway in the improvement of IR by ES were investigated. A total of 70 Sprague-Dawley male rats were divided into five groups: Normal $(n=10)$, IR control $(n=15)$, diet $(n=15)$, ES $(n=15)$ and $E S+$ diet $(n=15)$ groups. An IR rat model was established by high-fat and high-carbohydrate diet for 5 weeks and confirmed by measurement of fasting plasma glucose (FPG), insulin, insulin sensitivity index (ISI) and IR index. ES on the Zusanli (ST36), Sanyinjiao (SP 6) and Weiwanxiashu (EX-B3) acupoints and the low-fat and low-carbohydrate diet demonstrated protective effects. The body weight, concentrations of FPG, insulin, triglycerides (TG), free fatty acids (FFA) and total cholesterol (TC) of the rats were detected. Pathologic changes in the liver and pancreatic tissues were assessed. Western blotting and immunohistochemistry were performed to determine the role of PI3K/Akt/mTOR signaling. Results demonstrated that ES and diet therapy significantly increased ISI and reduced FPG, IR index, FFA, TG, TC and weight. Inflammatory cell infiltration in the liver and pancreatic tissues was ameliorated and lipid droplets and cavitation in hepatocyte were decreased after ES and diet therapy. The administration of ES and diet therapy also enhanced glucose transport by the upregulation of glucose transporter 4 and accelerated glycogen synthesis
\end{abstract}

Correspondence to: Professor Cheng-Lin Tang, Traditional Chinese Medicine College, Chongqing Medical University, 1 Yixue Road, Yuzhong, Chongqing 400016, P.R. China

E-mail: tangchenglin@cqmu.edu.cn

Abbreviations: IR, insulin resistance; ES, electrical stimulation; mTOR, mammalian target of rapamycin; FPG, fasting plasma glucose; ISI, insulin sensitivity index; TG, triglycerides; FFA, free fatty acids; TC, total cholesterol

Key words: insulin resistance, PI3K/Akt/mTOR signaling, electrical stimulation, diet therapy, glucose transport, glycogen synthesis through the suppression of glycogen synthase kinase $3 \alpha / \beta$ via PI3K/Akt/mTOR signaling. Hence, the present results demonstrated that ES combined with diet therapy improved IR through PI3K/Akt/mTOR signaling. The proposed therapy was superior to the method of diet alone.

\section{Introduction}

Insulin resistance (IR) is an impaired insulin response, which can cause damage to target organs. IR causes impaired glucose tolerance, as in diabetes mellitus and cardiovascular disease (1-3). A number of conventional pharmacological strategies are used to treat IR. However, limitations, such as costs, patients' compliance and drug side effects (including weight gain, hypoglycemia and congestive heart failure) are involved $(4,5)$. Thus, methods to enhance insulin sensitivity have become a topic of interest.

Multiple studies have demonstrated that electrical stimulation (ES) can influence the absorption, distribution and excretion of substances, and even induce the secretion of endogenous opioid peptides from multiple sites $(6,7)$. Therefore, plasma glucose levels are decreased. In addition to its hypoglycemic action, ES also improves insulin sensitivity by lowering free fatty acid (FFA) levels and increasing plasma insulin-like growth factor 1 levels, thereby regulating insulin secretion (8). Although ES has a hypoglycemic effect, ES treatment combined with other methods are required to improve therapeutic outcomes in clinical setting. The exact mechanism of ES on IR is not completely understood because of the complex pathological factors in IR (4).

$\mathrm{PI} 3 \mathrm{~K}$, Akt and mammalian target of rapamycin (mTOR) signaling are the main pathways that transduce insulin signaling $(9,10)$. Under normal conditions, insulin receptor substrate-1 (IRS-1) is phosphorylated and recruited by the tyrosine kinase, an insulin receptor (11). Then, the downstream PI3K is triggered, which generates phosphatidylinositol (3-5)-triphosphate and recruits Akt to the plasma membrane (11). Sufficient Akt, which phosphorylates the downstream target glycogen synthase kinase-3 (GSK-3), is recruited to stimulate the insulin response for glycogen synthesis (12). Akt also serves as a highly-regulated point of divergence for glucose transport by accelerating glucose transporter 4 (GLUT4) (13). If excessive calories are consumed, then the levels of toxic lipids and proinflammatory cytokines in the blood will be enhanced. Hyperglycemia 
upregulates the activity of $\mathrm{mTOR} / \mathrm{p} 70$ ribosomal S6 protein kinase (p70S6K) (14), which stimulates IRS-1 Ser636/639 phosphorylation and suppresses IRS-1-associated PI3K/Akt signaling, and GSK-3 is activated (14). Glycogen synthesis and glucose transport are impaired.

These previous studies have demonstrated that mTOR signaling is an important effector mediating the action of insulin. In theory and in clinic application, ES has an effect on IR, but only a few studies have examined this possibility $(6,7)$. Additional studies are necessary to investigate the molecular and intracellular mechanisms by which ES regulates and improves IR. The present study identified that ES can increase insulin sensitivity and improve IR by regulating PI3K, Akt and mTOR signaling. In the current study, IR models were established by a high fat and high carbohydrate diet (HFHCD). Then, the IR rats were treated by ES and diet therapy for 2 weeks. Hepatic and pancreas islet pathological changes and insulin-related signaling pathways were examined to investigate whether a combination of ES and diet therapy can improve insulin activity in rats and to explore the mechanism underlying the function of ES and diet therapy. The results suggested a theoretical basis for the clinical application of ES to treat patients with obesity or diabetes having IR.

\section{Materials and methods}

Animals. A total of 70 Sprague-Dawley male 4-week-old rats weighing 90-100 g were provided by the Experimental Animal Center of Chongqing Medical University (medical animal certification no. SCXK-2012-0002). The approval for the present study was provided by Chongqing Medical University's Animal Care and Ethics Committee and formulated by the Ministry of Science and Technology of China. The rats were housed in a room under controlled temperature and humidity $\left(24^{\circ} \mathrm{C}, 50 \%\right)$ with a $12 \mathrm{~h}$ light/dark cycle. The rats were provided with food and water ad libitum. After 1 week, the rats were randomly divided into normal $(n=10)$ and IR $(n=60)$ groups. The HFHCD composition and preparation described in a previous study was used with a few modifications (15). The HFHCD consisted of $25 \%$ fructose, $15 \%$ lard oil, $60 \%$ powdered rat chow and $50 \mathrm{~g}$ water per $\mathrm{kg}$ of diet. The IR rats were fed with HFHCD for 5 weeks. On the 6 th week, the IR state was confirmed by measuring fasting plasma glucose (FPG) insulin ( $\geq 100 \mathrm{mg} / \mathrm{dl})$, insulin sensitivity index (ISI) and IR index. All IR rats that satisfied the IR criteria were randomly divided into 4 groups, as follows: IR control group $(n=15)$, in which the rats were fed HFHCD continuously; diet group $(n=15)$, in which the rats were only fed a low-fat and low-carbohydrate diet (10\% fructose, $5 \%$ lard oil, $85 \%$ powdered rat chow and $50 \mathrm{~g}$ water per $\mathrm{kg}$ of diet); ES group $(n=15)$, in which the rats received ES and normal diet; and $\mathrm{ES}+$ diet group $(\mathrm{n}=15)$, in which the rats received low-fat and low carbohydrate diet and ES. All IR rats were fed for an additional 2 weeks on the experimental diets after the 5 weeks of the HFHCD and were subsequently sacrificed for the tissue collection.

ES application. The rats were positioned in a custom-made holder (patent no. ZL201420163074.2). ES was performed in bilateral Zusanli (ST36, located at the anterior aspect of the hind limb, $2 \mathrm{~mm}$ straight below the knee joint), Sanyinjiao (Spleen 6, located at the medial side of the hind limb, $2 \mathrm{~mm}$ above the tip of the medial malleolus posterior to the medial border of the tibia) and Weiwanxiashu (EX-B3, $1 \mathrm{~mm}$ lateral to the no. 8 thoracic spinous process) acupoints after the IR model was established. Acupuncture needles (13 $\mathrm{mm}$ in length and $0.22 \mathrm{~mm}$ in diameter) were inserted into the acupoints muscle layers to a depth of $\sim 5 \mathrm{~mm}$. The pairs of needles on the ipsilateral ST36 and SP6 acupoints were connected with the output terminals of an ES apparatus (Model SDZ-II; Suzhou Huatuo Medical Instruments Co., Ltd.). The alternating strings of dense-sparse frequencies $(20 \mathrm{~Hz}$ for $1.05 \mathrm{sec}$ and $4 \mathrm{~Hz}$ for $2.85 \mathrm{sec}$, alternately) were used. The intensity was adjusted to induce a slight twitch of the hind limbs ( $\leq 2 \mathrm{~mA})$, with intensity lasting for $20 \mathrm{~min} /$ day for 14 days.

Reagents. Glucose oxidase kit (Nanjing Jiancheng Taihao Biotechnology Co., Ltd. cat. no. F006) and INS ELISA kit (Thermo Fisher Scientific, cat. no. ERINS) were used. The primary antibodies were used for western blotting and immunohistochemistry as follows: Polyclonal rabbit anti-P70S6K antibody (Signalway Antibody LLC, cat. no. 21276), polyclonal rabbit anti-GSK-3 $\alpha / \beta$ antibody (Signalway Antibody LLC, cat. no. 29039), polyclonal rabbit anti-mTOR antibody (Signalway Antibody LLC, cat. no. 21214), monoclonal rabbit anti-Akt Antibody (Signalway Antibody LLC, cat. no. 48888), monoclonal rabbit anti-PI3K antibody (Signalway Antibody LLC, cat. no. 48868) and polyclonal rabbit anti-GLUT4 antibody (Abcam, cat. no. ab654). The secondary antibody for western blotting and immunohistochemistry: Goat anti-rabbit IgG secondary antibody (OriGene Technologies, Inc. cat. no. TA130015).

Tissue processing for ultrastructure observation and immunohistochemistry. The rats from the various groups were anesthetized by intraperitoneally injecting $2 \%$ pentobarbital sodium $(40 \mathrm{mg} / \mathrm{kg})$. Physiological saline $\left(250 \mathrm{ml}, 4^{\circ} \mathrm{C}\right)$ and $4 \%$ paraformaldehyde in $500 \mathrm{ml}$ of $0.1 \mathrm{mmol} / 1$ phosphate buffered saline (PBS; $4^{\circ} \mathrm{C}, \mathrm{pH}$ of 7.4 ) were perfused into the left ventricle. The liver tissues, pancreas and the quadriceps femoris muscle tissues in left leg were harvested and assigned into two segments. First segments were fixed with $4 \%$ paraformaldehyde for $24-48 \mathrm{~h}\left(4^{\circ} \mathrm{C}\right)$ and dehydrated with $30 \%$ sucrose overnight $\left(4^{\circ} \mathrm{C}\right)$. Second segments were used for ultrastructure observation.

Hematoxylin-eosin $(H \& E)$ staining. To evaluate the pathologic changes and effectiveness of ES, H\&E staining was performed. Liver and pancreas tissues were fixed in formalin for $>24 \mathrm{~h}$ and then moved to $70 \%$ ethanol. Then, the tissues were dehydrated in graded alcohols, immersed in xylol and embedded in paraffin wax. The tissues were sliced into serial sections with a thickness of $6 \mu \mathrm{m}$ each using a paraffin slicing machine and stained with H\&E (0.5-1\% eosin; 3.3\% hematoxylin) under room temperature for $80 \mathrm{~min}$. Digital images were captured using an Olympus light microscope with a x10 objective and an Olympus camera (Olympus-45; Olympus Corporation) with a total magnification of $x 100$. The images were further analyzed by a blinded investigator. 
Immunohistochemical analysis. For the immunohistochemical evaluation, $10 \mu \mathrm{m}$ thick sections of the liver, pancreas and muscle tissues from each group were prepared. Briefly, endogenous peroxidase was blocked by $3 \% \mathrm{H}_{2} \mathrm{O}_{2}$ for $15 \mathrm{~min}$ at room temperature. The sections were further washed with $0.01 \mathrm{mmol} / \mathrm{l}$ PBS and blocked with $10 \%$ horse serum (Thermo Fisher Scientific, Inc., cat. no. 31874) for $20 \mathrm{~min}$ at room temperature. Next, the sections were incubated with primary antibodies (PI3K, Akt, GLUT 4, GSK-3 $\alpha$, GSK-3 $\beta$, mTOR, P70S6K; all 1:200) at $4^{\circ} \mathrm{C}$ overnight. The sections were rinsed with $0.01 \mathrm{mmol} / \mathrm{l} \mathrm{PBS}$ and reacted with secondary antibodies (1:400) for $20 \mathrm{~min}$ at $37^{\circ} \mathrm{C}$. The slides were rewashed with $0.01 \mathrm{mmol} / \mathrm{l} \mathrm{PBS}$. DAB horseradish peroxidase color development kit (Beyotime Institute of Biotechnology, cat. no. P0202) was used for developing at room temperature for 5-10 min. The slides were counterstained by $3.3 \%$ hematoxylin at room temperature for 2 min after the slides were rewashed with $0.01 \mathrm{mmol} / \mathrm{l}$ PBS. Lastly, the slides were mounted in $50 \%$ glycerol dissolved in $0.01 \mathrm{mmol} / \mathrm{l} \mathrm{PBS}$.

Ultrastructure observation. A portion of each specimen from the sections perfused with $4 \%$ paraformaldehyde was immediately fixed in $2.5 \%$ glutaraldehyde for 2 days at room temperature after washing with PBS. The glutaraldehyde-fixed specimens were treated in $2 \%$ osmic acid for $1 \mathrm{~h}$, dehydrated in serial alcohol (50\% ethanol, $75 \%$ ethanol, $95 \%$ ethanol and absolute alcohol), infiltrated with propylene oxide and embedded in araldite for morphometric analyses on semithin sections (70-90 nm). The sections were mounted on $\mathrm{Cu}$ grids and stained with $3 \%$ uranyl acetate for $30 \mathrm{~min}$ and then $2 \%$ lead citrate for $15 \mathrm{~min}$ at room temperature. Images were obtained by transmission electron microscopy (Hitachi-7500; Hitachi, Ltd.).

Measurement ofmetabolic parameters FPG, insulin, FFA, total cholesterol (TC) and triglycerides (TG). Blood was collected from the left cardiac cavity and centrifuged at 3,000 $\mathrm{x} \mathrm{g}$ for $10 \mathrm{~min}$ at $4^{\circ} \mathrm{C}$ to isolate the supernatant/plasma. FPG levels were determined using a glucose oxidase kit according to the manufacturer's protocol. The plasma levels of the fasting insulin were measured by INS ELISA kit (Thermo Fisher Scientific, Inc., cat. no. ERINS). Non-esterified free fatty acids assay kit (Nanjing Jiancheng Bioengineering Institute, cat. no. A042-2-1), total cholesterol assay kit (Nanjing Jiancheng Bioengineering Institute, cat. no. F002-1-1) and triglyceride assay kit (Nanjing Jiancheng Bioengineering Institute, cat. no. A110-1-1) were used to measure FFA, TC and TG, respectively. The ISI was defined as follows: ISI=l g (1/FPG x serum insulin). The IR index was defined as follows: IR index $=F P G$ level $(\mathrm{mg} / \mathrm{dl}) \mathrm{x}$ fasting serum insulin level $(\mathrm{ng} / \mathrm{ml}) / 22.5(16)$.

Western blot analysis. Protein extraction was performed according to standard procedures. Briefly, samples were homogenized in $0.3 \mathrm{ml}$ of disrupting RIPA lysis buffer (Beyotime Institute of Biotechnology, cat. no. P0013B) and 1\% protease inhibitor (Beyotime Institute of Biotechnology, cat. no. ST506). The homogenate was centrifuged at $12,000 \mathrm{x} g$ for $15 \mathrm{~min}$ at $4^{\circ} \mathrm{C}$. The supernatant was diluted with $5 \mathrm{X}$ protein loading buffer (Beyotime Institute of Biotechnology) and then heated at $95^{\circ} \mathrm{C}$ for $5 \mathrm{~min}$. Samples containing $40 \mu \mathrm{g}$ protein were separated by $12 \%$ SDS-PAGE and then transferred onto a polyvinylidene difluoride membrane. Blotted membranes were blocked with 5\% skimmed milk at room temperature for $4 \mathrm{~h}$. For immunoblotting, the following primary antibodies were used: Monoclonal rabbit anti-PI3 kinase antibody $(1: 1,000)$, monoclonal rabbit anti-Akt antibody $(1: 1,000)$, polyclonal rabbit anti-GLUT4 antibody $(1: 1,000)$, polyclonal rabbit anti-GSK-3 $\alpha / \beta$ antibody $(1: 1,000)$, polyclonal rabbit anti-P70S6K antibody $(1: 1,000)$ and polyclonal rabbit anti-mTOR antibody $(1: 1,000)$. All the primary antibodies were incubated at $4^{\circ} \mathrm{C}$ overnight. The secondary antibody used was goat anti-rabbit $\operatorname{IgG}(1: 500)$ at room temperature for $2 \mathrm{~h}$. Western bands were quantified by gel densitometry (GS-900 ${ }^{\mathrm{TM}}$ calibrated densitometry system, cat. no. 1707991, Bio-Rad Laboratories, Inc.). The ratio between protein and GAPDH expression for each sample was obtained (each point was repeated in triplicate).

Statistical analysis. The statistical analyses were performed using SPSS 11.0 (SPSS, Inc.). The results were expressed as the mean \pm standard error of the mean. Data were compared using the Duncan Multiple Range test following one-way ANOVA. $\mathrm{P}<0.05$ was considered to indicate a statistically significant difference.

\section{Results}

Metabolic parameters and weight. After being fed with HFHCD for 5 weeks, the rats demonstrated signs of IR; however, no significant changes in overall body weight were observed in the IR rats compared with the normal rats (Table I). Following the administration of specific diets, the weight of rats in the diet, ES and ES + diet group were significantly decreased compared with those in the IR control group $(\mathrm{P}<0.05$; Table I). The weight of rats in the $\mathrm{ES}+$ diet group significantly decreased compared with in the diet and ES groups $(\mathrm{P}<0.05$; Table I). This demonstrated that ES combined with low-carbohydrate, low-fat diet can reduce weight significantly. The HFHCD caused IR, as indicated by a significant increase in FPG, insulin, IR index, TG, FFA and TC, and a decrease in ISI compared with the normal group (Table I). After 14 days, the rats in the ES + diet group demonstrated significantly lower serum levels of FPG, insulin, IR index, TG, FFA and TC compared with those in the IR control, diet and ES groups $(\mathrm{P}<0.05, \mathrm{n}=15$; Table $\mathrm{I})$.

Pathologic changes in liver tissue and pancreatic islet. As demonstrated in Fig. 1, hepatocytes in the normal group were distributed around the central veins radially and presented cord-like arrangement. Hepatocellular nuclei were clear without inflammation and necrosis. HFHCD induced considerable hepatic damage with evident necrotic foci and inflammatory cell infiltration. The hepatic cord structure in the IR control group was destroyed and the hepatocyte arrangement became disorderly (Fig. 1). Considerable lipid droplets and cavitations were observed in hepatocytes by H\&E staining. The diet treatment resulted in insignificant changes compared with the IR control group. The ES-treated group demonstrated fewer fat droplets, whereas the steatosis in the livers of the rats in the ES + diet group was improved (Fig. 1). These observations were confirmed by electron microscopy 
(Fig. 1), which revealed hepatocytes with abundant lipid droplets in the IR control group and few fat droplets in the diet group. In the ES and ES + diet group, inflammatory cell infiltration was improved. Lipid droplets and cavitations in the hepatocyte decreased. The hepatocyte arrangement in the ES + diet group was notably enhanced compared with that in the IR control group. These results suggested that treatment with ES and diet could ameliorate the effects of HFHCD.

The H\&E-stained sections revealed that the pancreatic islet in the normal group was oval and large compared with the IR control group, thereby indicating that the pancreatic islet was injured. The pancreatic islet was improved in the ES + diet group compared with the other two treatment groups. This result suggested that ES facilitated the maintenance of the pancreatic islet cell morphology.

PI3K/Akt/mTOR signaling in muscle or hepatic tissues is regulated by ES and diet therapy. To investigate whether $\mathrm{PI} 3 \mathrm{~K} / \mathrm{Akt} / \mathrm{mTOR}$ signaling was involved with the mechanism of ES and diet therapy in insulin activity in rats, mTOR, p70S6K, PI3K and Akt expression levels were examined at the lesion sites in the different groups by immunohistochemistry and western blot analysis. As demonstrated in Fig. 2, the protein expression levels of mTOR and its downstream substrate, p70S6K, were significantly increased in the muscle tissue of IR rats fed with HFHCD ( $\mathrm{P}<0.05$; Fig. 2). Following treatment, the protein expression levels of mTOR and p70S6K significantly decreased in the ES + diet group compared with the other groups $(\mathrm{P}<0.05$; Fig. 2$)$.

$\mathrm{PI} 3 \mathrm{~K}$ and Akt protein expression levels were analyzed in the hepatic tissues. Western blot analysis demonstrated that the $\mathrm{PI} 3 \mathrm{~K}$ and Akt expression levels significantly decreased in the IR rats compared with the normal rats $(\mathrm{P}<0.05)$ (Fig. $3 \mathrm{C}$ and $\mathrm{D})$. This result was consistent with the immunohistochemistry results (Fig. 3A and B). Following treatment, the PI3K and Akt expression levels were upregulated in the ES + diet group compared with rats in other groups $(\mathrm{P}<0.05$; Fig. 3$)$. These results suggested that the excessive nutrients hyperactivated the mTOR pathway and may have led to IR via suppression of $\mathrm{PI} 3 \mathrm{~K} / \mathrm{Akt}$ signaling. This result was consistent with previous findings (14). ES and diet treatment may have improved IR by mTOR inhibition and PI3K/Akt signaling activation.

ES downregulates GSK-3 $\alpha / \beta$ and upregulates GLUT4 expression. The protein expression levels of GSK-3 $\alpha$ in liver tissue and GSK-3 $\beta$ in muscle tissue, which are serine kinases and identified as key negative regulators of glycogen synthesis (17), significantly increased in the IR control group compared with the normal groups (Fig. 4). Following treatment, the GSK-3 $\alpha / \beta$ expression levels in the ES + diet group were significantly downregulated compared with the other groups (Fig. 4; P<0.05). Thus, ES may improve glycogen synthesis and IR by inhibiting GSK-3 $\alpha / \beta$ expression (18). To detect the effect of ES and diet therapy on glycogen synthesis, the protein expression levels of GLUT4, which maintains glucose homeostasis in striated muscle (19), were also investigated. The results demonstrated that the GLUT4 protein expression levels increased significantly in the quadriceps femoris muscle tissues of the ES + diet group compared with those of the other groups (Fig. 5; $\mathrm{P}<05$ ). This result suggested that combination 


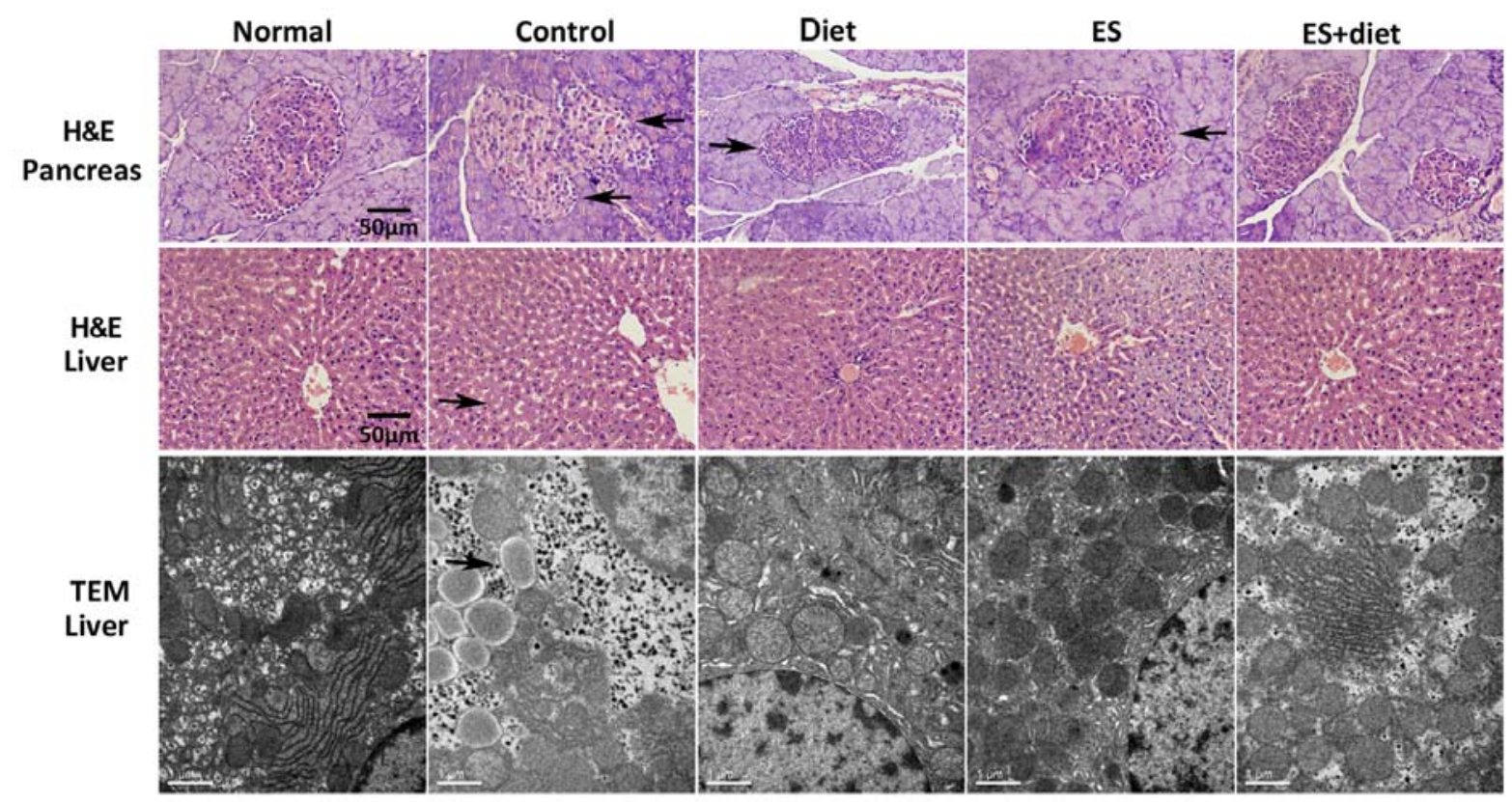

Figure 1. Pathologic changes in liver tissue and pancreatic islet. Representative images from H\&E stained sections of pancreas and liver tissues are shown for each experimental group. In addition, representative images are shown from TEM analysis of liver sections. Arrows point to lipid droplets. H\&E, haemotoxylin and eosin; TEM, transmission electron microscopy; ES, electrical stimulation.

treatment may improve glucose transportation by enhancing GLUT4 expression.

\section{Discussion}

Dietary excesses, including high fat and hyperglycemia, increase the toxic lipid and proinflammatory cytokine levels in the blood, which are major risk factors for chronic inflammation and IR development in the skeletal muscle $(20,21)$. The prevalence of IR disorders is mainly associated with obesity, type 2 diabetes mellitus, dyslipidemia and hypertension in western/westernized populations (22). IR includes impaired fatty acid oxidation, lipotoxicity and ectopic fat deposition (23), whereas lipotoxicity and ectopic fat are the common features of the diverse IR states (24).

Feeding rats with HFHCD induces IR (25). The duration of HFHCD feeding in the literature varies predominantly from 4-12 weeks $(25,26)$. In the present study, the IR rat models were established by HFHCD for 5 weeks. The increased FPG, insulin and IR index and decreased ISI in rats indicated the successful establishment of the IR model. The FFA, TG and TC concentrations in the control IR group increased. These findings were consistent with those of previous studies, which demonstrated that hyperglycemia induces IR, vascular dysfunction and hepatic steatosis in rats $(25,27)$.

Hepatic steatosis caused by TG accumulation is a major contributor to IR (28). This phenomenon was confirmed in the rat liver specimens of the present study by histopathological examination (Fig. 1). mTOR is one of the intermediates produced during TG synthesis and acts as an inhibitor for insulin signaling (29). Hyperglycemia upregulates the activity of the mTOR/p70S6K axis (14). Increased mTOR/p70S6K1 affects insulin signaling by the phosphorylation of insulin receptor substrate-1, thereby inhibiting PI3K and Akt activation $(30,31)$. The serine/threonine protein kinase Akt, which is also known as protein kinase $\mathrm{B}$ and is a downstream effector of PI3K, is a critical mediator of mTOR activity $(32,33)$. In the present study, HFHCD-induced IR was associated with increased mTOR and decreased PI3K and Akt protein levels (Figs. 2 and 3).

Skeletal muscle is the main target tissue of insulin action (13). At the cellular level, IR in the skeletal muscle is characterized by impaired insulin stimulation of glucose uptake and glycogen synthesis $(34,35)$. The promotion of glucose transport and uptake and glycogen synthesis are the key biological actions of insulin in skeletal muscle (13).

A number of studies have proved the important role of ES in hypoglycemic action in IR $(8,36-38)$. However, the true effect and mechanism of ES on IR is unclear and remains controversial. The results of the present study demonstrated that ES can accelerate glucose translocation and glycogen synthesis to improve IR by regulating the PI3K/Akt/mTOR signaling pathway. This model may provide a mechanistic explanation for the findings. Following the combination of ES and diet treatment, FFA, TG and TC concentrations were significantly decreased and inflammatory cell infiltration in the liver and the pancreatic islet were ameliorated compared with the other groups. mTOR, which acts as an inhibitor for insulin signaling, was increased by HFHCD (29) and attenuated by ES and diet treatment. In addition to the skeletal muscle, hepatic tissue is also a main target tissue of insulin action (13). The levels of PI3K and Akt proteins in the liver were regulated by ES and diet treatment.

In the further downstream signaling from Akt, the insulin-mediated translocation of GLUT4 starts from its intracellular localization to the plasma membrane for glucose transport (13). GLUT4 in the striated muscle and adipose cells is the major glucose transporter in maintaining glucose homeostasis $(24,25)$. Insulin stimulates glucose transport by promoting GLUT4 exocytosis (13). The impaired glucose transport activity and/or defective intracellular metabolism of 
A

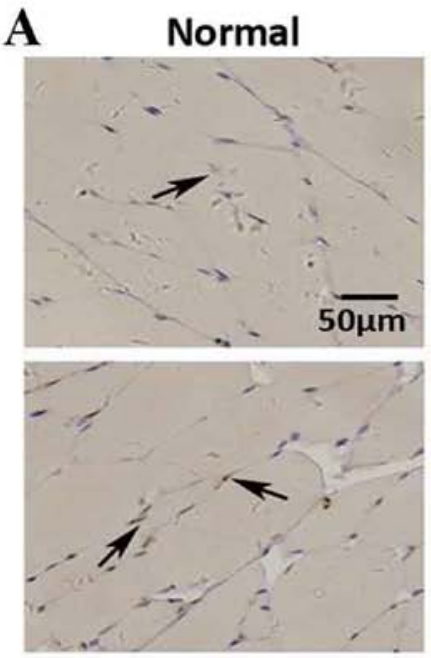

ES

B
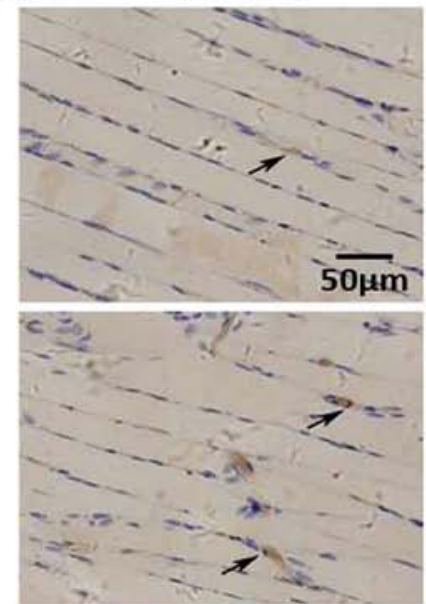

ES
Control
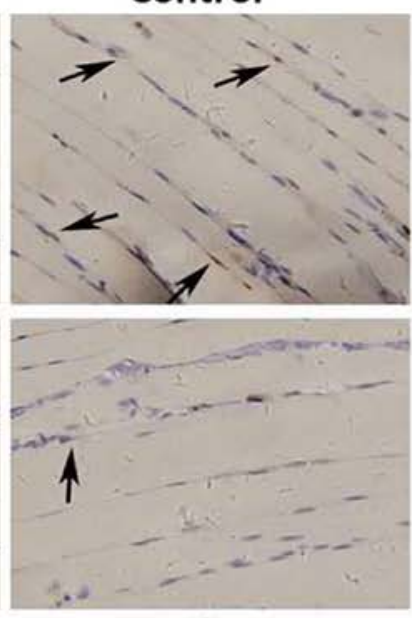

ES+diet

Control
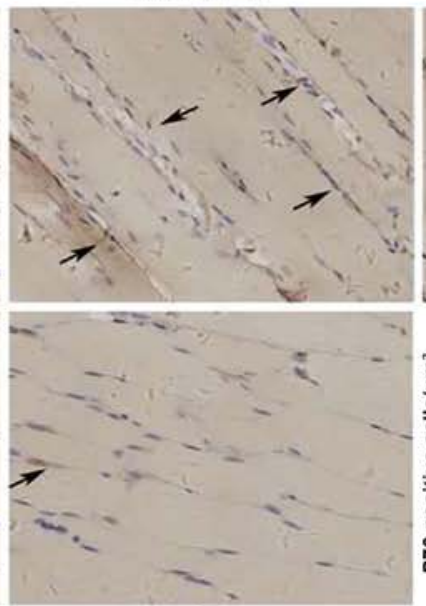

ES+diet

.
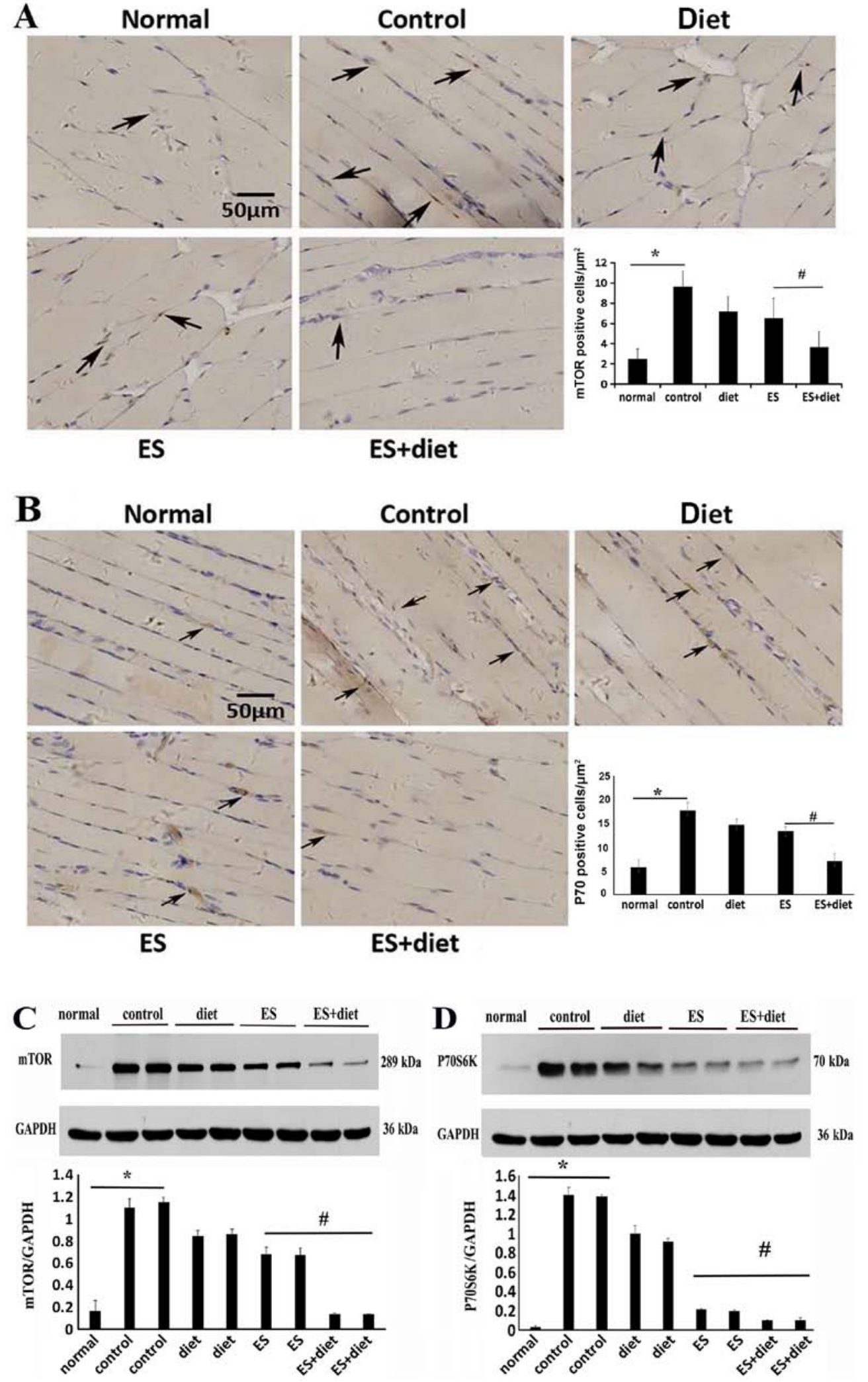

Figure 2. mTOR and P70S6K expression levels in muscle tissues from the different groups. (A) Immunohistochemistry analysis of mTOR (arrows show mTOR-immunoreactive cells) and (B) p70S6K protein expression (arrows show p70S6K-immunoreactive cells). Scale bar, 50 $\mu$ m. (C) Western blot analysis of mTOR and (D) p70S6K protein expression levels. GAPDH was used as an internal control. Data are presented as the mean \pm standard error of the mean. ${ }^{\mathrm{P}}<0.05 \mathrm{vs}$. normal group; ${ }^{\#} \mathrm{P}<0.05$ vs. ES group ( $\mathrm{n}=10$ per group). mTOR, mammalian target of rapamycin; p70S6K, p70 ribosomal S6 protein kinase; ES, electrical stimulation.

the transported glucose can decrease glucose uptake (19). This result is consistent with the present study, showing that the decreased protein levels for GLUT4 in IR rats induced the increased fasting glucose. In rodents and humans, the skeletal muscle is responsible for $>80 \%$ of insulin-mediated glucose uptake in vivo through muscle contraction (26), which induces 


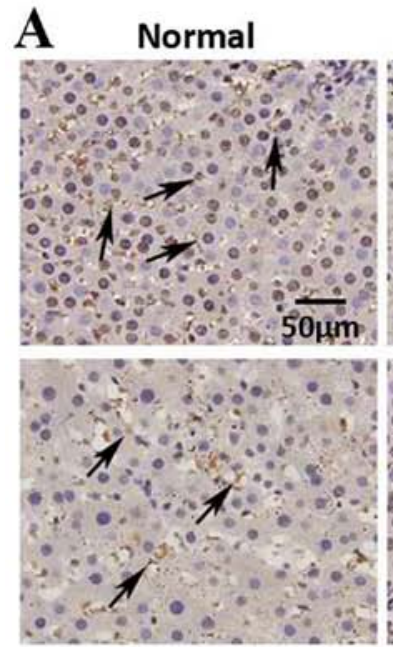

ES

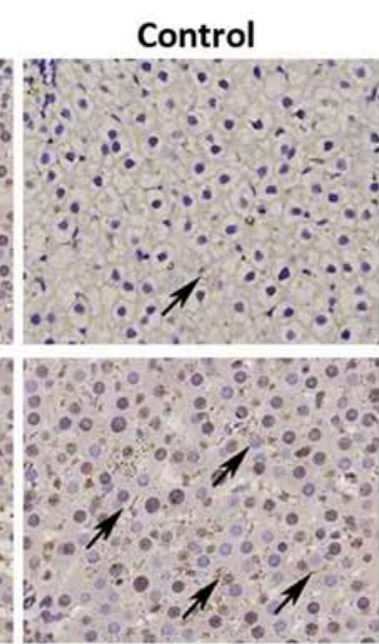

ES+diet
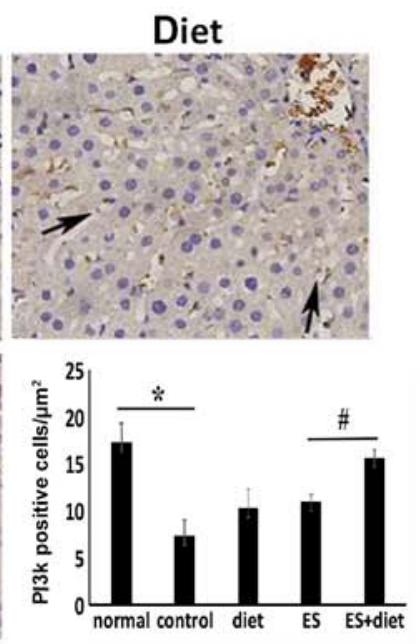

B Normal

Control

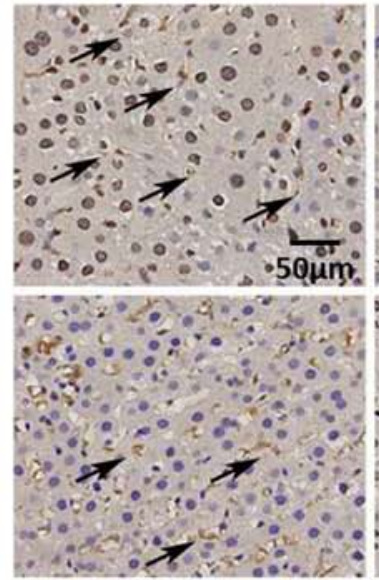

ES
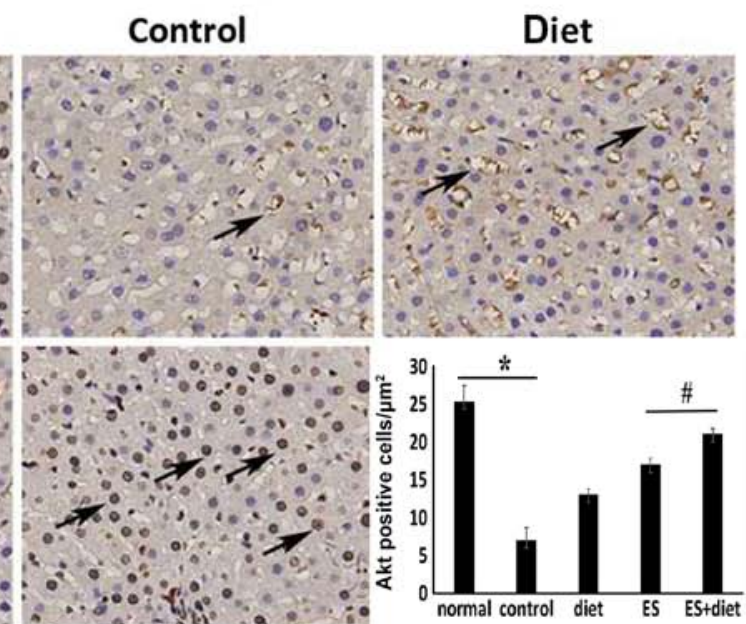

ES+diet
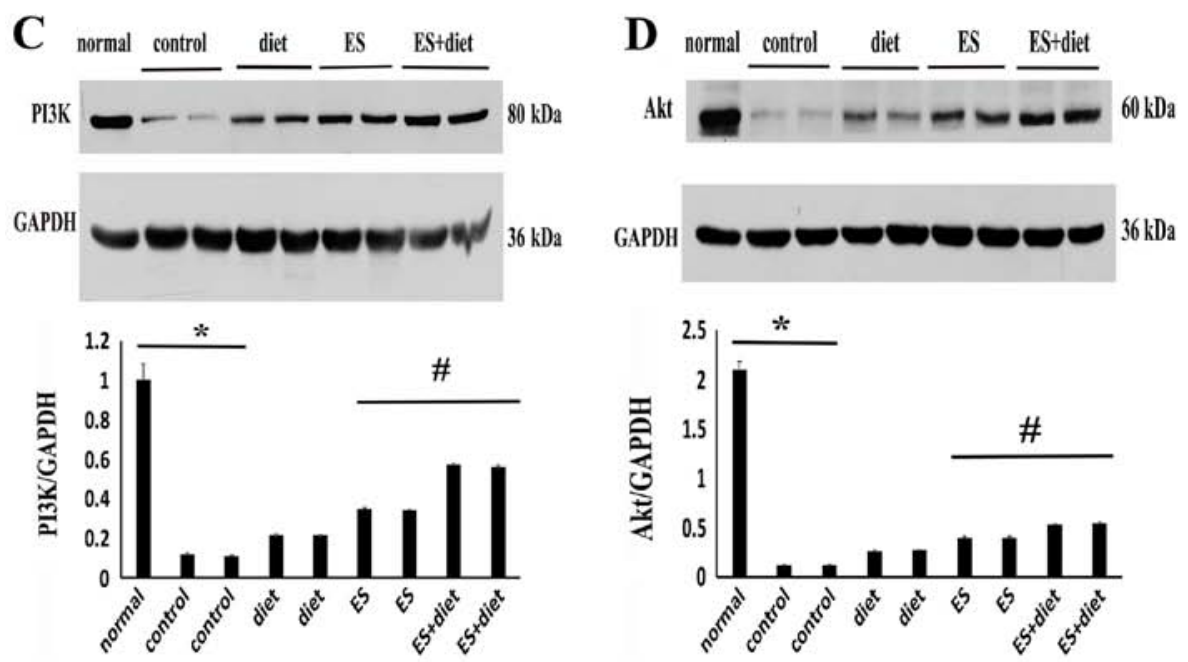

Figure 3. PI3K and Akt expression levels in hepatic tissues from the different groups. (A) Immunohistochemistry analysis of PI3K (arrows show p70S6K-immunoreactive cells) and (B) Akt protein expression (arrows show Akt-immunoreactive cells). Scale bar, $50 \mu \mathrm{m}$. (C) Western blot analysis of PI3K and (D) Akt protein expression levels. GAPDH was used as an internal control. Data are presented as mean \pm standard error of the mean. ${ }^{*} \mathrm{P}<0.05$ vs. normal group; ${ }^{\#} \mathrm{P}<0.05$ vs. ES group ( $n=10$ per group). ES, electrical stimulation.

GLUT4 translocation from the intracellular depots to the plasma membrane to allow large transport of glucose (23). Only a moderate increase in the protein levels of GLUT4 is required to enhance insulin sensitivity after exercise in skeletal muscle (27). In the present study, alternating strings of low and dense-sparse frequencies used in the ES + diet group may have 
A

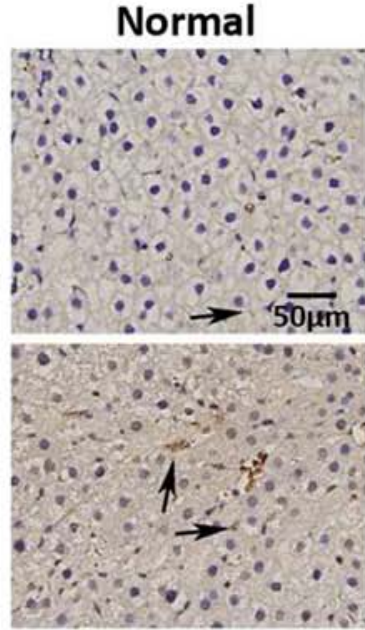

ES

B
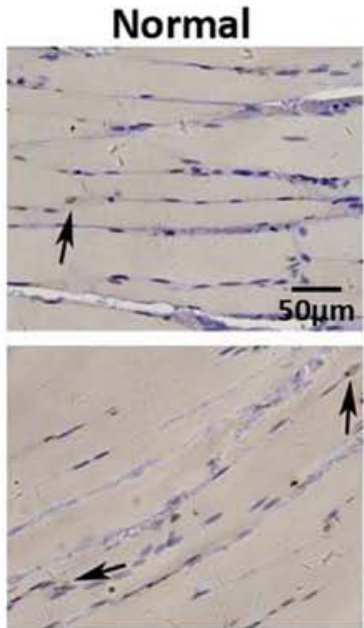

ES
Control

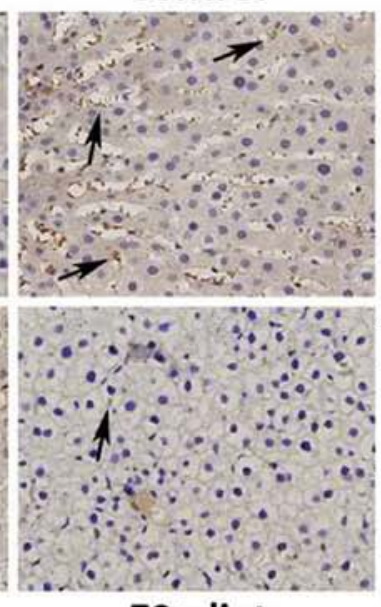

ES+diet

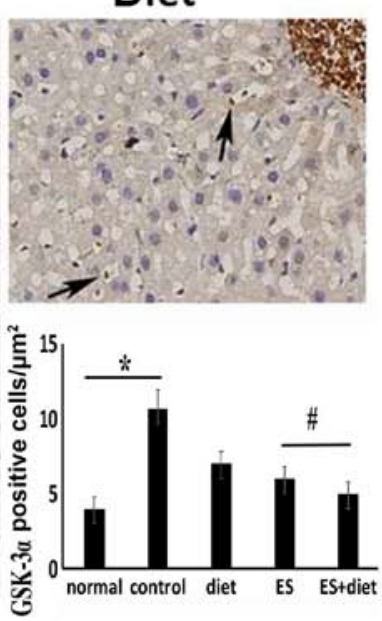

Diet

Control
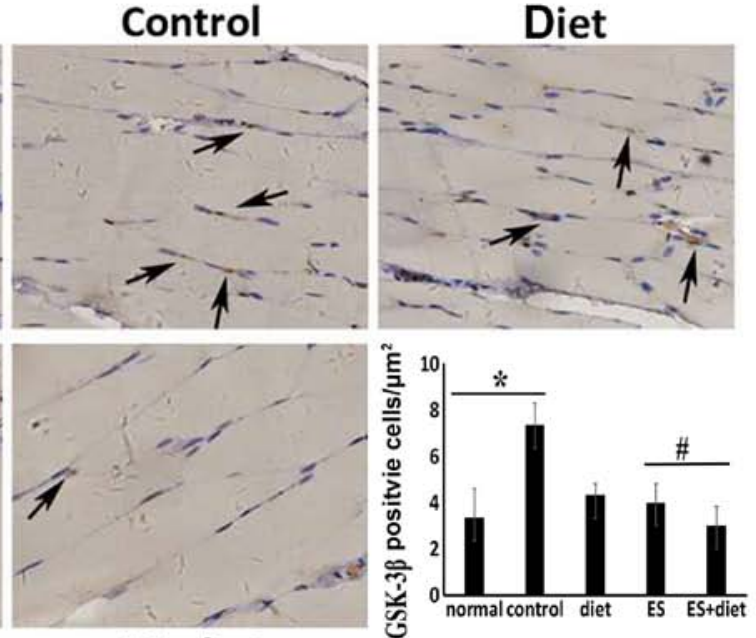

ES+diet
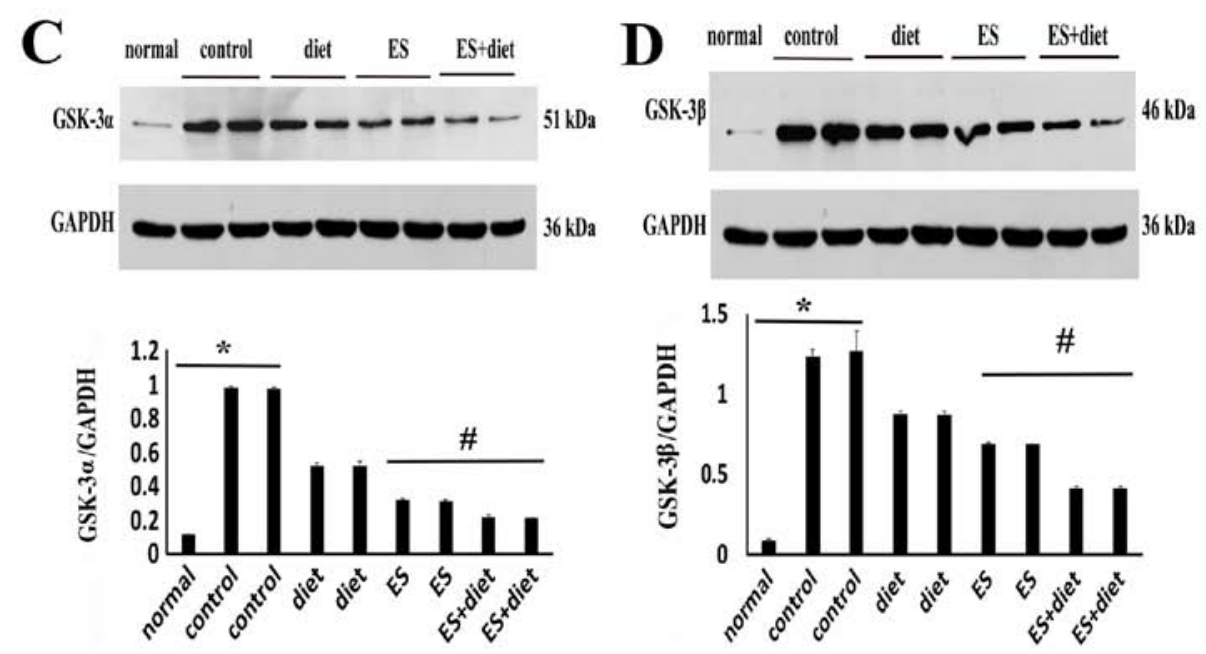

Figure 4. GSK-3 $\alpha$ and GSK-3 $\beta$ expression in the hepatic and muscle tissues, respectively, from the different groups. (A) Immunohistochemistry analysis of GSK-3 $\alpha$ (arrows show GSK-3 $\alpha$-immunoreactive cells) and (B) GSK-3 $\beta$ protein expression GSK-3 $\beta$ (arrows show GSK-3 $\beta$-immunoreactive cells). Scale bar, $50 \mu \mathrm{m}$. (C) Western blot analysis of GSK-3 $\alpha$ and (D) GSK-3 $\beta$ protein expression levels. GAPDH was used as an internal control. Data are presented as mean \pm standard error of the mean. ${ }^{*} \mathrm{P}<0.05$ vs. normal group. ${ }^{\#} \mathrm{P}<0.05$ vs. ES group ( $\mathrm{n}=10$ per group). GSK-3, glycogen synthase kinase 3 ; ES, electrical stimulation.

stimulated skeletal muscle contraction and induced glucose transportation by increasing PI3K, Akt and GLUT4 protein levels. ES combined with diet also increased GLUT4 protein levels, which may improve glucose transport and uptake. 

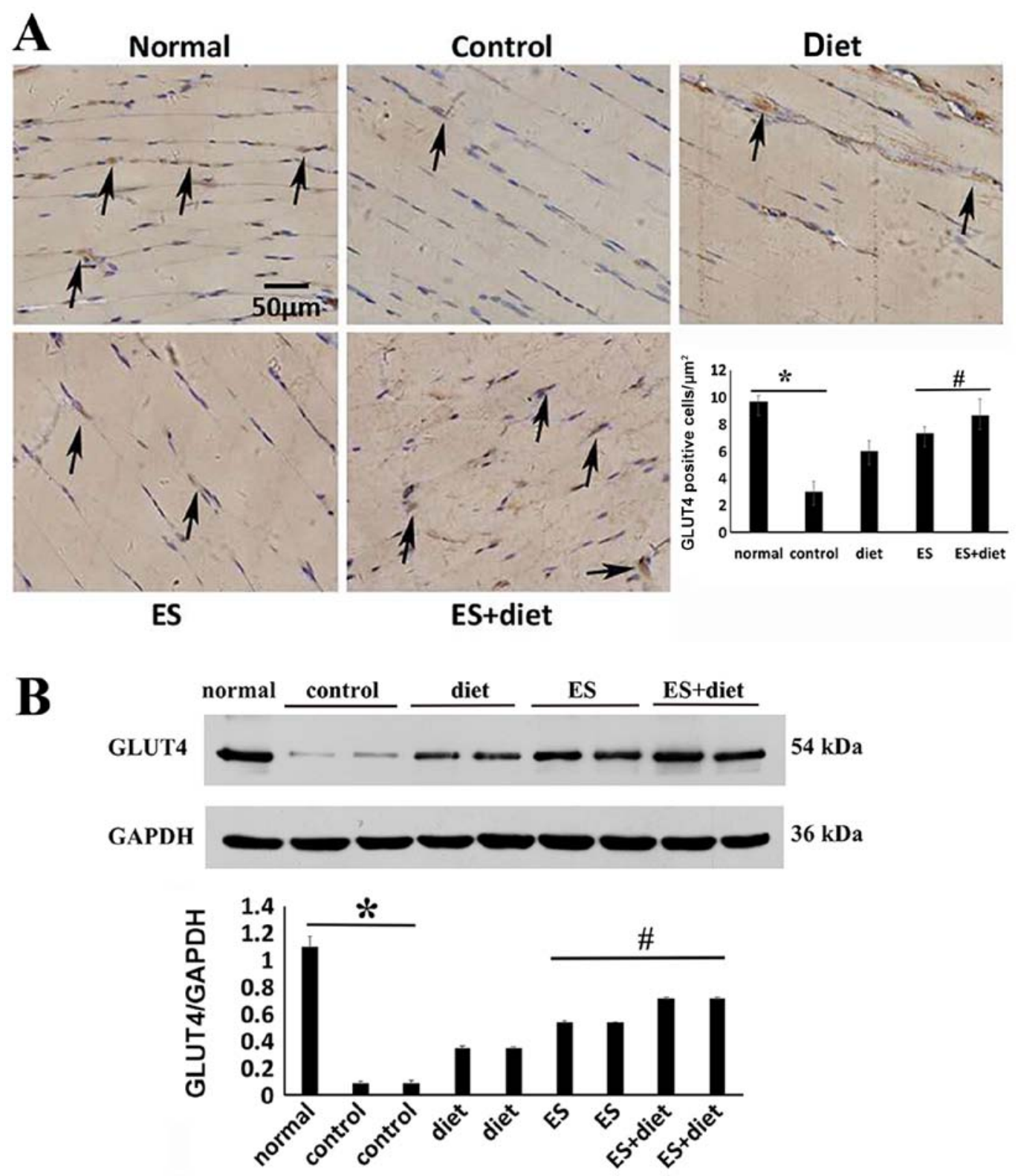

Figure 5. GLUT4 expression levels in muscle tissues from the different groups. (A) Immunohistochemistry analysis of GLUT4 protein expression (arrows show GLUT4-immunoreactive cells). Scale bar, $50 \mu \mathrm{m}$. (B) Western blot analysis of GLUT4 protein expression levels. GAPDH was used as an internal control. Data are presented as mean \pm standard error of the mean. ${ }^{*} \mathrm{P}<0.05$ vs. normal group. ${ }^{\#} \mathrm{P}<0.05$ vs. ES group (n=10 per group). GLUT4, glucose transporter 4 ; ES, electrical stimulation.

In addition to glucose transport, ES and diet therapy may improve glycogen synthesis by inhibiting GSK3, which includes GSK-3 $\alpha / \beta$ and is involved in IR (17). GSK-3 is a key negative regulator of glycogen synthesis, which is a major form of glucose storage $(28,29)$. GSK-3 $\alpha$ functions as the hepatic glycogen synthesis kinase regulating glycogen synthesis and deposition primarily in the liver. GSK-3 $\beta$ serves an important role in the skeletal muscle tissue and $\beta$-islet cells where its knockout leads to enhanced glycogen synthesis activity and glycogen accumulation or insulin responsiveness, respectively (39). Excessive calorie intake enhances IRS-1 Ser636/639 phosphorylation, suppresses IRS-1-associated PI3K/Akt signaling and activates GSK-3 $\alpha$ and GSK-3 $\beta$ (14). Thus, insulin signaling is impaired. The current study demonstrated that Akt decreased, whereas GSK- $3 \alpha / \beta$ protein levels in the liver and skeletal muscle tissue increased in the IR control groups, respectively. Following combination treatment, Akt was activated by ES, and GSK-3 $\alpha$ and GSK-3 $\beta$ were inhibited. These findings suggested that the effects of ES on GSK-3 $\alpha$ and GSK-3 $\beta$ may contribute to improving glucose homeostasis.

The present study proposed a mechanism for the effect of ES on IR. The results indicated that ES and diet therapy improved insulin sensitivity in the IR rats via mTOR signaling. ES increased GLUT4 expression and inhibited GSK-3 expression, which may result in regulating glucose transport and glycogen synthesis in the skeletal muscle and liver tissue, respectively. The present in vivo data supplied the theoretical basis for the clinical use of ES to treat patients with obesity or diabetes having IR. However, these mechanisms have not been fully elucidated. Thus, the molecular consequences of insulin-mediated changes in mTOR signaling in vitro should be established in future studies. 


\section{Acknowledgements}

Not applicable.

\section{Funding}

The present study was supported by the National Natural Science Foundation of China (grant nos. 81403466 and 81273870), the Natural Science Foundation Project of CQ CSTC (grant nos. cstc2017jcyjAX0363 and cstc2018jcyjAX0036), the Joint Project of CQ CSTC and Health Commission of Chongqing (grant no. ZY201802026) and the Venture \& Innovation Support Program for Chongqing Overseas Returnees (grant no. cx2018106).

\section{Availability of data and materials}

The datasets used and/or analyzed in the present study are available from the corresponding author on reasonable request.

\section{Authors' contributions}

$\mathrm{SH}$ was involved in data acquisition, analysis and interpretation, as well as manuscript drafting and revision. NT and $\mathrm{HZ}$ were involved in data acquisition and analysis and manuscript drafting. CT was involved in the conception and design of the study and gave final approval of the manuscript to be submitted. All authors read and approved the final manuscript.

\section{Ethics approval and consent to participate}

The approval for the present study was provided by Chongqing Medical University's Animal Care and Ethics Committee and formulated by the Ministry of Science and Technology of China.

\section{Patient consent for publication}

Not applicable.

\section{Competing interests}

The authors declare that they have no competing interests.

\section{References}

1. Crescenzo R, Bianco F, Mazzoli A, Giacco A, Liverini G and Iossa S: Mitochondrial efficiency and insulin resistance. Front Physiol 5: 512, 2015

2. Anusree SS, Nisha VM, Priyanka A and Raghu KG: Insulin resistance by TNF- $\alpha$ is associated with mitochondrial dysfunction in 3T3-L1 adipocytes and is ameliorated by punicic acid, a PPAR $\gamma$ agonist. Mol Cell Endocrinol 413: 120-128, 2015.

3. Kawaguchi T, Nakano D, Oriishi T and Torimura T: Effects of isomaltulose on insulin resistance and metabolites in patients with non-alcoholic fatty liver disease: A metabolomic analysis. Mol Med Rep 18: 2033-2042, 2018.

4. Lin RT, Tzeng CY, Lee YC, Chen YI, Hsu TH, Lin JG and Chang SL: Acupoint-specific, frequency-dependent, and improved insulin sensitivity hypoglycemic effect of electroacupuncture applied to drug-combined therapy studied by a randomized control clinical trial. Evid Based Complement Alternat Med 2014: 371475, 2014.
5. Lee TI, Bai KJ, Chen YC, Lee TW, Chung CC, Tsai WC, Tsao SY and Kao YH: Histone deacetylase inhibition of cardiac autophagy in rats on a high-fat diet with low-dose streptozotocin-induced type 2 diabetes mellitus. Mol Med Rep 17: 594-601, 2018.

6. Lin JG, Chen WC, Hsieh C, Tsai CC, Cheng YW, Cheng JT and Chang SL: Multiple sources of endogenous opioid peptide involved in the hypoglycemic response to $15 \mathrm{~Hz}$ electroacupuncture at the Zhongwan acupoint in rats. Neurosci Lett 366: 39-42, 2004.

7. Senna-Fernandes V, Franca D, Moreno SF, Santos-Filho S, Rogers PA, Bernardo-Filho M and Guimarães MA: The effect of 'Zusanli' (ST. 36) acupuncture on the bio-availability of sodium pertechnetate in Wistar rats. Acupunct Electrother Res 31: 33-44, 2006.

8. Lee YC, Li TM, Tzeng CY, Cheng YW, Chen YI, Ho WJ, Lin JG and Chang SL: Electroacupuncture-induced cholinergic nerve activation enhances the hypoglycemic effect of exogenous insulin in a rat model of streptozotocin-induced diabetes. Exp Diabetes Res 2011: 947138, 2011.

9. Könner AC and Brüning JC: Selective insulin and leptin resistance in metabolic disorders. Cell Metab 16: 144-152, 2012.

10. Cheng L, Song J, Li G, Liu Y, Wang Y, Meng X, Sun G and Sun X: Effects of the Tangningtongluo formula as an alternative strategy for diabetics via upregulation of insulin receptor substrate-1. Mol Med Rep 16: 703-709, 2017.

11. Guo S, Copps KD, Dong X, Park S, Cheng Z, Pocai A, Rossetti L, Sajan M, Farese RV and White MF: The Irs1 branch of the insulin signaling cascade plays a dominant role in hepatic nutrient homeostasis. Mol Cell Biol 29: 5070-5083, 2009.

12. Sarbassov DD, Guertin DA, Ali SM and Sabatini DM: Phosphorylation and regulation of Akt/PKB by the rictor-mTOR complex. Science 307: 1098-1101, 2005.

13. Tzatsos A and Kandror KV: Nutrients suppress phosphatidylinositol 3-kinase/Akt signaling via raptor-dependent mTOR-mediated insulin receptor substrate 1 phosphorylation. Mol Cell Biol 26: 63-76, 2006.

14. Panchal SK, Poudyal H, Iyer A, Nazer R, Alam A, Diwan V, Kauter K, Sernia C, Campbell F, Ward L, et al: High-carbohydrate high-fat diet-induced metabolic syndrome and cardiovascular remodeling in rats. J Cardiovasc Pharmacol 57: 611-624, 2011.

15. Nozaki Y, Fujita K, Wada K, Yoneda M, Shinohara Y, Imajo K, Ogawa Y, Kessoku T, Nakamuta M, Saito S, et al: Deficiency of eNOS exacerbates early-stage NAFLD pathogenesis by changing the fat distribution. BMC Gastroenterol 15: 177, 2015.

16. Leng S, Zhang W, Zheng Y, Liberman Z, Rhodes CJ, Eldar-Finkelman $\mathrm{H}$ and Sun XJ: Glycogen synthase kinase 3 beta mediates high glucose-induced ubiquitination and proteasome degradation of insulin receptor substrate 1. J Endocrinol 206 : 171-181, 2010.

17. Nikoulina SE, Ciaraldi TP, Mudaliar S, Carter L, Johnson K and Henry RR: Inhibition of glycogen synthase kinase 3 improves insulin action and glucose metabolism in human skeletal muscle. Diabetes 51: 2190-2198, 2002.

18. Kahn BB, Rossetti L, Lodish HF and Charron MJ: Decreased in vivo glucose uptake but normal expression of GLUT1 and GLUT4 in skeletal muscle of diabetic rats. J Clin Invest 87: 2197-2206, 1991.

19. Zhang L, Keung W, Samokhvalov V, Wang W and Lopaschuk GD: Role of fatty acid uptake and fatty acid beta-oxidation in mediating insulin resistance in heart and skeletal muscle. Biochim Biophys Acta 1801: 1-22, 2010.

20. Zeyda M and Stulnig TM: Obesity, inflammation, and insulin resistance-a mini-review. Gerontology 55: 379-386, 2009.

21. Sparks LM, Xie H, Koza RA, Mynatt R, Hulver MW, Bray GA and Smith SR: A high-fat diet coordinately downregulates genes required for mitochondrial oxidative phosphorylation in skeletal muscle. Diabetes 54: 1926-1933, 2005.

22. McGarry JD: Banting lecture 2001: Dysregulation of fatty acid metabolism in the etiology of type 2 diabetes. Diabetes 51: 7-18, 2002.

23. Lund S, Holman GD, Schmitz O and Pedersen O: Contraction stimulates translocation of glucose transporter GLUT4 in skeletal muscle through a mechanism distinct from that of insulin. Proc Natl Acad Sci USA 92: 5817-5821, 1995.

24. James DE, Strube M and Mueckler M: Molecular cloning and characterization of an insulin-regulatable glucose transporter. Nature 338: 83-87, 1989. 
25. Zorzano A, Wilkinson W, Kotliar N, Thoidis G, Wadzinkski BE, Ruoho AE and Pilch PF: Insulin-regulated glucose uptake in rat adipocytes is mediated by two transporter isoforms present in at least two vesicle populations. J Biol Chem 264: 12358-12363, 1989.

26. DeFronzo RA, Jacot E, Jequier E, Maeder E, Wahren J and Felber JP: The effect of insulin on the disposal of intravenous glucose. Results from indirect calorimetry and hepatic and femoral venous catheterization. Diabetes 30: 1000-1007, 1981.

27. Ivy JL and Kuo CH: Regulation of GLUT4 protein and glycogen synthase during muscle glycogen synthesis after exercise. Acta Physiol Scand 162: 295-304, 1998.

28. Grimes CA and Jope RS: The multifaceted roles of glycogen synthase kinase 3beta in cellular signaling. Prog Neurobiol 65: 391-426, 2001.

29. Laplante $M$ and Sabatini DM: mTOR signaling in growth control and disease. Cell 149: 274-293, 2012.

30. Kaidanovich-Beilin $\mathrm{O}$ and Eldar-Finkelman $\mathrm{H}$ : Long-term treatment with novel glycogen synthase kinase-3 inhibitor improves glucose homeostasis in ob/ob mice: Molecular characterization in liver and muscle. J Pharmacol Exp Ther 316: 17-24, 2006.

31. Pearce NJ, Arch JR, Clapham JC, Coghlan MP, Corcoran SL, Lister CA, Llano A, Moore GB, Murphy GJ, Smith SA, et al: Development of glucose intolerance in male transgenic mice overexpressing human glycogen synthase kinase-3beta on a muscle-specific promoter. Metabolism 53: 1322-1330, 2004.

32. Sun XJ and Liu F: Phosphorylation of IRS proteins Yin-Yang regulation of insulin signaling. Vitam Horm 80: 351-387, 2009.

33. Taniguchi CM, Emanuelli B and Kahn CR: Critical nodes in signalling pathways: Insights into insulin action. Nat Rev Mol Cell Biol 7: 85-96, 2006.
34. Højlund $\mathrm{K}$ and Beck-Nielsen $\mathrm{H}$ : Impaired glycogen synthase activity and mitochondrial dysfunction in skeletal muscle: Markers or mediators of insulin resistance in type 2 diabetes? Curr Diabetes Rev 2: 375-395, 2006.

35. Shulman GI, Rothman DL, Jue T, Stein P, DeFronzo RA and Shulman RG: Quantitation of muscle glycogen synthesis in normal subjects and subjects with non-insulin-dependent diabetes by $13 \mathrm{C}$ nuclear magnetic resonance spectroscopy. N Engl J Med 322: 223-228, 1990.

36. Chang SL, Lin KJ, Lin RT, Hung PH, Lin JG and Cheng JT Enhanced insulin sensitivity using electroacupuncture on bilateral Zusanli acupoints (ST 36) in rats. Life Sci 79: 967-971, 2006.

37. Pai HC, Tzeng CY, Lee YC, Chang CH, Lin JG, Cheng JT and Chang SL: Increase in plasma glucose lowering action of rosiglitazone by electroacupuncture at bilateral Zusanli acupoints (ST.36) in rats. J Acupunct Meridian Stud 2: 147-151, 2009.

38. Pan H, Huang H, Zhang L, Ma S, Yang H and Wang H: 'Adjusting internal organs and dredging channel' electroacupuncture treatment prevents the development of diabetic peripheral neuropathy by downregulating glucose-related protein 78 (GRP78) and caspase-12 in streptozotocin-diabetic rats. J Diabetes, Mar 8 , 2019 (Epub ahead of print).

39. MacAulay K and Woodgett JR: Targeting glycogen synthase kinase-3 (GSK-3) in the treatment of Type 2 diabetes. Expert Opin Ther Targets 12: 1265-1274, 2008.

(i) $($ ) This work is licensed under a Creative Commons Attribution-NonCommercial-NoDerivatives 4.0 International (CC BY-NC-ND 4.0) License. 